\title{
The influence of transverse slope effects on large scale morphology in morphodynamic mod- els
}

\author{
Anne Baar ${ }^{1, *}$, Marcio Boechat Albernaz ${ }^{1}$, Wout van Dijk ${ }^{1}$, and Maarten Kleinhans ${ }^{1}$ \\ ${ }^{1}$ Department of Physical Geography, Utrecht University, Utrecht, The Netherlands
}

\begin{abstract}
Large-scale morphology is greatly affected by the amount of downslope sediment transport on slopes transverse to the main flow direction, which determines bar length and bifurcation dynamics. Consequently, the transverse slope parameter is a crucial part of morphodynamic models. However, existing models have the tendency to overpredict channel depth and braiding index, and therefore slope effects are often increased when calibrating on existing morphology. The objective of this study is to identify possible causes of the overdeepening of channels in the morphodynamic model Delft3D, and to show how different slope effect parameterisations affect morphology in rivers and estuaries. Results show that the two methods to calculate transverse sediment transport in Delft3D have a significantly different effect on the predicted morphology and this effect is larger for environments with a large braiding index, in combination with the sediment transport predictor of Engelund-Hansen. Results furthermore imply that even when models are initially calibrated on existing morphology, results could significantly differ downstream when calibrated with a different choice of transverse slope option.
\end{abstract}

\section{Introduction}

Present morphodynamic models are sensitive to a calibration of the transverse bed slope parameter, which determines the length of fluvial bars and active channel width [1], braiding index [2,3], stability of river bifurcations [4], and wave lengths of coastal sandbanks and sandwaves $[5,6]$. The transverse slope parameter determines the amount of downslope sediment transport due to gravity on slopes transverse to the main flow direction. When secondary currents are present, downslope sediment transport due to gravity is balanced by helical flows dragging the sediment upslope, and thereby also controls the adaptation of the bed to perturbations in the flow. An incorrect setting thus has major consequences for the predicted large-scale morphology, bank protection works and dredging volumes for fairway maintenance.

However, current morphodynamic models tend to underestimate slope effects, and thereby over-predict channel depth and braiding index, and therefore slope effects are often artificially increased when calibrating on existing morphology [1, 7]. Transverse slope

*e-mail: a.w.baar@uu.nl 
predictors in these models are simple functions of sediment mobility, and based on previous studies that isolated a specific transport mechanism or bed state and studied its separate effect on the transverse slope. Consequently, existing transverse slope predictors were validated with a small series of experiments with a limited range in flow characteristics and sediment mobility depending on the process that was studied. However, these predictors are now used in numerical morphodynamic modelling where all processes act in combination. Baar et al. [8] performed 224 experiments with a large range in bend flow conditions and sediment sizes, resulting in transverse slopes from almost horizontal to the angle of repose, and bed states ranging from a lower-stage plane bed, across the ripple-dune threshold, to an upper-stage plane bed. They showed that the realistic calibration range of the slope parameters is much smaller than current model studies imply, and suggest that such calibration is necessary to compensate for other, yet unidentified, model weaknesses such as issues with numerical schemes or missing processes.

The objective of this study is therefore to identify the cause of the overdeepening of channels in the morphodynamic model Delft3D, and to quantify the sensitivity of predicted longterm morphology to the transverse bed slope parameters, even when a measured bathymetry is used for calibration. To this end, we study the difference in equilibrium morphology between the two commonly used options to calculate the deflection of sediment transport on transverse slope: the method by Ikeda [9] (Islope = 2) and the method by Koch and Flokstra [10] (Islope $=3$ ). First, we will explain how slope effects are calculated in Delft3D, and indicate the main difference between both methods to calculate this downslope sediment transport. Then, we will describe the three models that are used is this study, and describe how they respond to changes in slope parameters.

\section{Model set-up}

\subsection{Slope effects in Delft3D}

The main difference between both slope options in Delft3D, is that the predictor of Ikeda [9] uses a critical shear stress, which is absent in the predictor of Koch and Flokstra [10]. However, also the resulting transport vector is calculated differently. Firstly, the magnitude of sediment transport is predicted, based on a situation of a flat bed with a single grainsize. Secondly, for Koch and Flokstra [10] the direction of sediment transport is corrected for transverse gradients by rotating the transport vector based on the user-defined factor $A_{s h}$ :

$$
\tan (\psi)=\frac{1}{A_{s h} \theta^{B_{s h}}} \frac{\partial z_{b}}{\partial y},
$$

while for Ikeda [9] an additional transport vector is calculated perpendicular to the flow direction, based on the input parameter $\alpha_{b n}$ :

$$
q_{n}=q_{s} \alpha_{b n} \sqrt{\frac{\theta_{c}}{\theta}} \frac{\partial z_{b}}{\partial y}
$$

where $\theta=$ sediment mobility $[-], q=$ sediment transport load $\left[\mathrm{m}^{2} / \mathrm{s}\right]$, and $\frac{\partial z_{b}}{\partial y}=$ transverse slope $[m / m]$.

As a result, the predictor of Ikeda [9] also increases the total magnitude of sediment transport when slope effects are increased on calibration, while for Koch and Flokstra [10] this is not influenced directly. The relation between $\alpha_{b n}$ and $A_{s h}$ is plotted in Fig. 1. The default value of $\alpha_{b n}$ in Delft3D is set to 1.5, while the parameter $A_{s h}$ is not defined in the model, but should be 1.5 according to Koch and Flokstra [10]. 


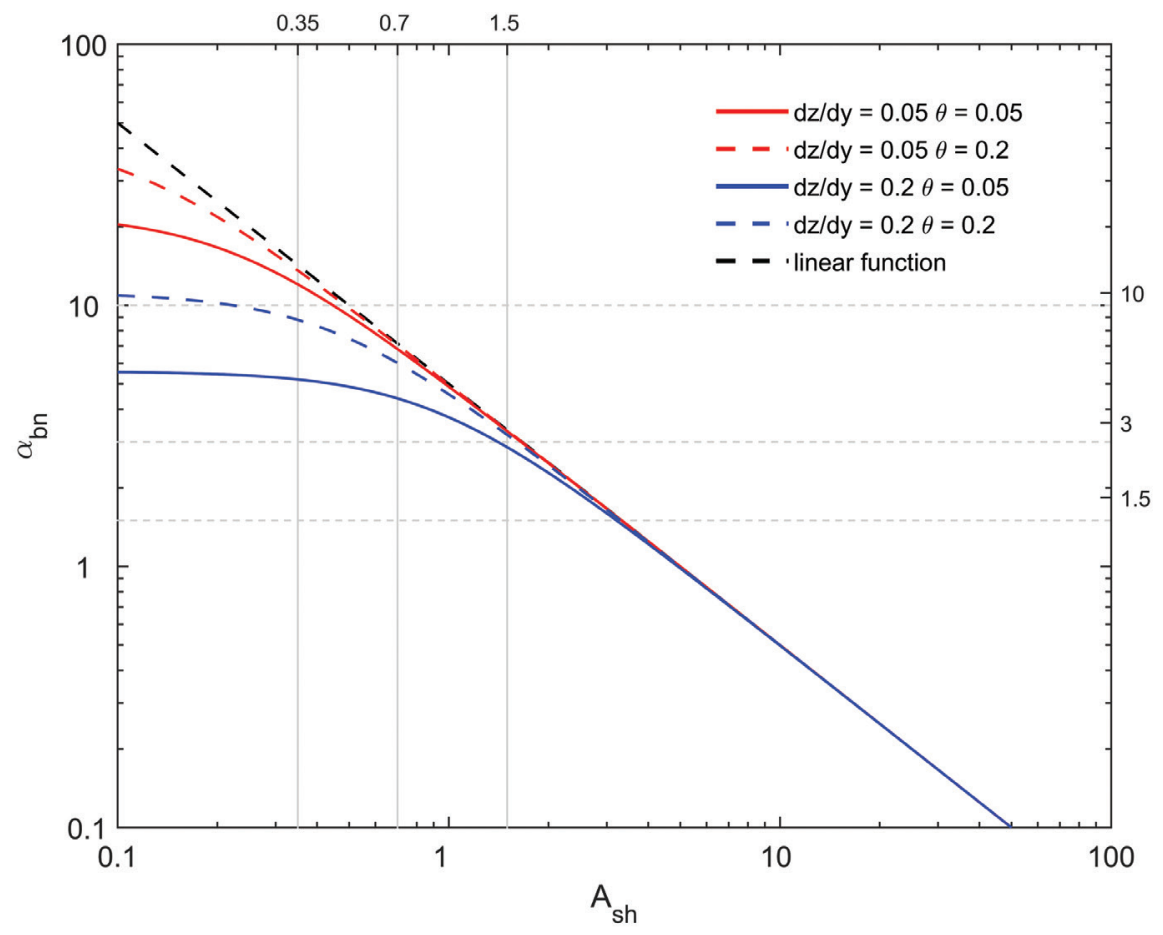

Figure 1. Relation between $\alpha_{b n}$ and $A_{s h}$ ([8]).

\subsection{Model runs}

To identify the cause of the overdeepening of channels in the morphodynamic model Delft3D, and to quantify the sensitivity of predicted long-term morphology to the transverse bed slope parameters, three different model studies are conducted. Firstly, a simple straight river channel of 5 grid cells wide is used to test several parameter settings and their effect on the balance of downslope sediment transport and channel incision. Secondly, the difference in effect of the two main transverse slope options is tested in an existing large-scale river model and in an existing large-scale estuary model. For all models, the depth-averaged version with parameterisation of secondary flow was used.

The 5 grid cell wide river model has a width of $200 \mathrm{~m}$ and a length of $5 \mathrm{~km}$, and a slope of $1 \mathrm{~mm} / \mathrm{m}$. the three rows in the middle have the same elevation, but the outer two rows of are $5 \mathrm{~m}$ higher so that they are above the water level. This way, the active river is three grid cells wide, but will not experience boundary effects of the model. A model of only three grid cells wide makes it easier to study the sediment fluxes between the cells and understand the equilibrium that will develop. The discharge is $2500 \mathrm{~m} 3 / \mathrm{s}$, divided over the 3 middle grid cells. The downstream boundary consists of a water level boundary. In this paper, we will only focus on the difference between equilibrium morphology when using the sediment transport predictors of Van Rijn [11](VR93), Van Rijn et al. [12](VR04), and Engelund and Hansen [13]. The default value of $\alpha_{b n}=1.5$ is used for slope effects.

The second model is the model from Schuurman et al. [1], who modelled a braided sandbed river. They varied the $A_{s h}$ between 0.35 and 1.5 with the sediment transport predictor of Engelund and Hansen [13], and concluded that an $A_{s h}$ of 0.7 is the optimal value for this 
system. We run the same models again with values of $\alpha_{b n}$ corresponding to their runs with varying $A_{s h}$, according to figure 1 . For example, their optimal value of 0.7 corresponds with an $\alpha_{b n}$ of 7. Furthermore, we run the model with realistic values according to the experimental results of Baar et al. [8] for both the $A_{s h}$ and the $\alpha_{b n}$.

The last model consists of a river delta influenced by tides, with the sediment transport predictor of Van Rijn et al. [12]. Here, we also use realistic values for both the $A_{s h}$ and the $\alpha_{b n}$, and test the effect of higher values for transverse sediment transport.

For both large-scale models, we determine the relative distribution of transverse slopes in the entire model, and compare these between model runs with different slope effects. For the river delta, we calculate sediment transport in both the streamwise and transverse direction, averaged over a cross-section on the river upstream and on the delta.

\section{Results}

\subsection{Simple model}

The results from the simple model show that different sediment transport predictors can have a different equilibrium between downslope sediment transport and channel incision (Fig. 2). When using the predictor of Engelund and Hansen [13], the three grid cells in the middle show no difference in sediment transport and the final equilibrium is the same as the initial morphology. However, the model with the sediment transport predictor of Van Rijn [11], which is the default option, shows a preference for the two outer cells of the active channel. Here, the transverse sediment transport does not balance the channel incision and two deep channels form. The middle cell ultimately fills up entirely. the model with the sediment transport predictor of Van Rijn et al. [12] shows a similar behavior, but chooses one cell to incise.

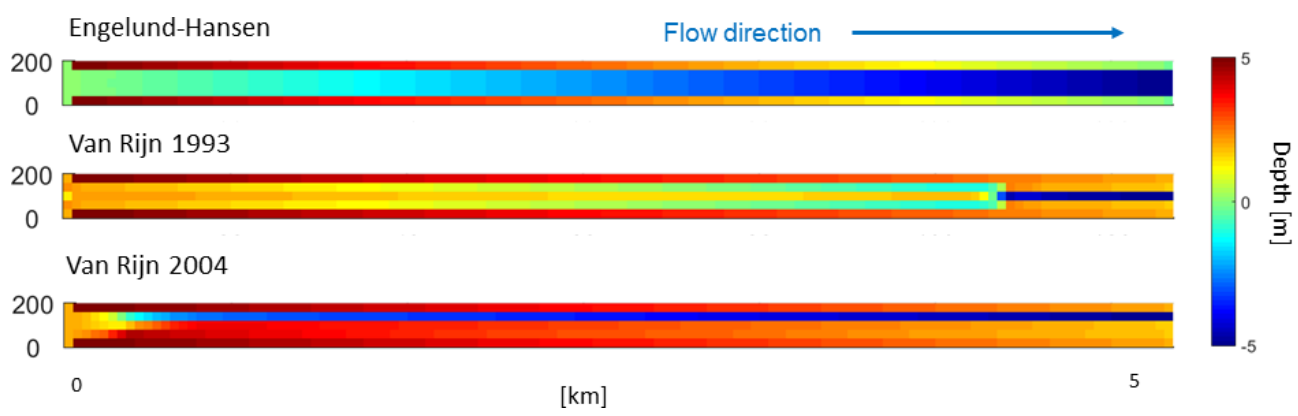

Figure 2. A simple straight river channel of 5 grid cells wide, run with three different sediment transport predictors.

\subsection{Large-scale morphology}

The braided river of Schuurman et al. [1] shows a large difference between both slope options (Fig. 3). The increase in total sediment transport results in a significant decrease in channel depth and braiding index compared to the original model runs. For example, the run with the optimal value of an $A_{s h}$ of 0.7 , as indicated by Schuurman et al. [1], shows a larger braiding index than the corresponding $\alpha_{b n}$ of 7, which has one larger and smoother channel. 
When modelling a river delta influenced by tides, and with the sediment transport predictor of Van Rijn et al. [12], results are less different between both slope options (Fig. 4). The river with the predictor of Koch and Flokstra [10] has a slightly deeper channel and a higher braiding index downstream than the model with the predictor of Ikeda [9]. This trend is visible for both the models with the realistic slope parameters (left side of Fig. 4), and the calibrated models (right side of Fig. 4). Both models with realistic slope parameters show unrealistically deep channels, as expected.

Interestingly, sediment transport, both in streamwise and in transverse direction, is in the same order of magnitude when averaged over the cross-section for models with corresponding slope effects. This means that, for corresponding $A_{s h}$ and $\alpha_{b n}$ values, the magnitude of sediment transport is indeed the same, but the direction is different. This difference in direction influences the location of sediment deposition, and thereby influences channel depth, bar length, and bifurcation dynamics downstream.

Figure 5 shows an overview of the slope distribution of all model runs. This figure illustrates that corresponding values for slope parameters result in different morphologies, but also that the differences are larger for the braided system. For the braided system, the values of $A_{s h}$ as used by Schuurman et al. [1] correspond with much lower values for $\alpha_{b n}$ when comparing resulting morphology using these slope distributions. For example, the optimal value of $A_{s h}$ of 0.7 corresponds with an $\alpha_{b n}$ of 1 instead of 7.

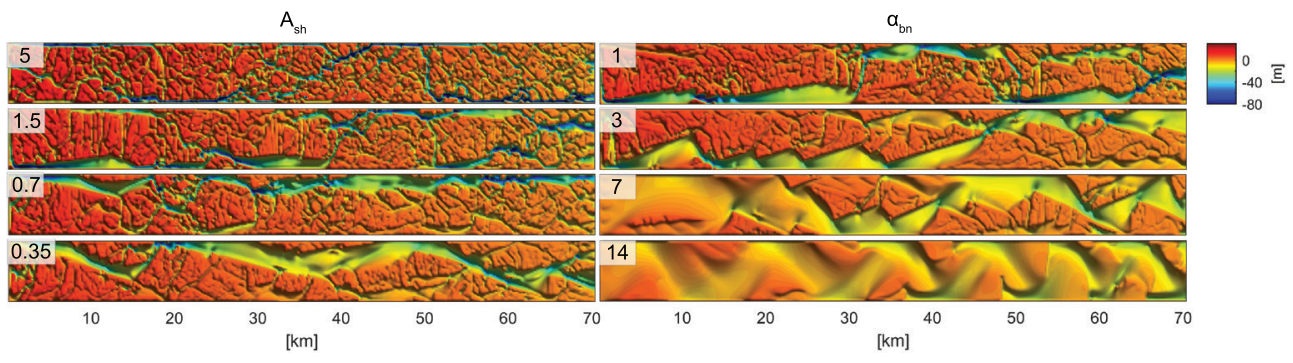

Figure 3. Braided river model of Schuurman et al. [1] with varying slope effects.
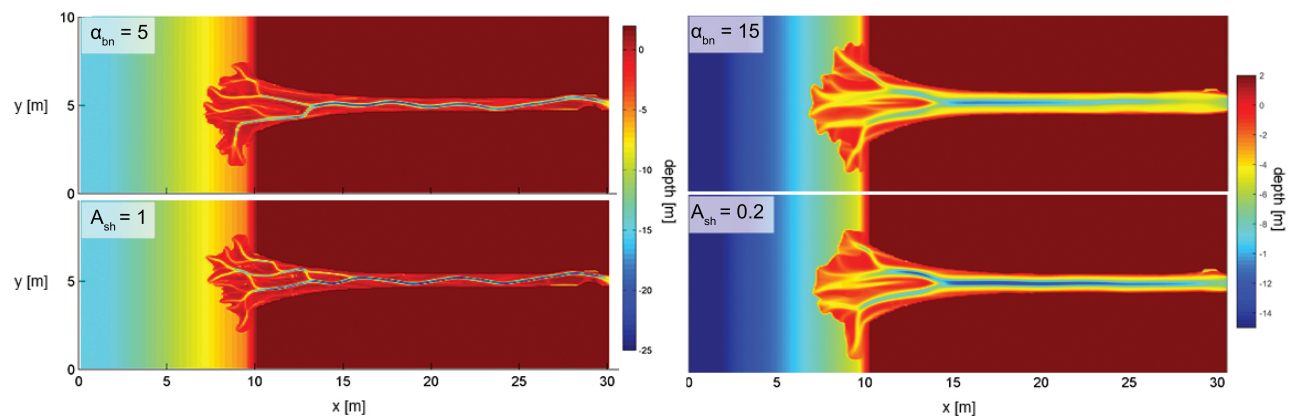

Figure 4. River delta with tidal influences, with varying slope effects. 


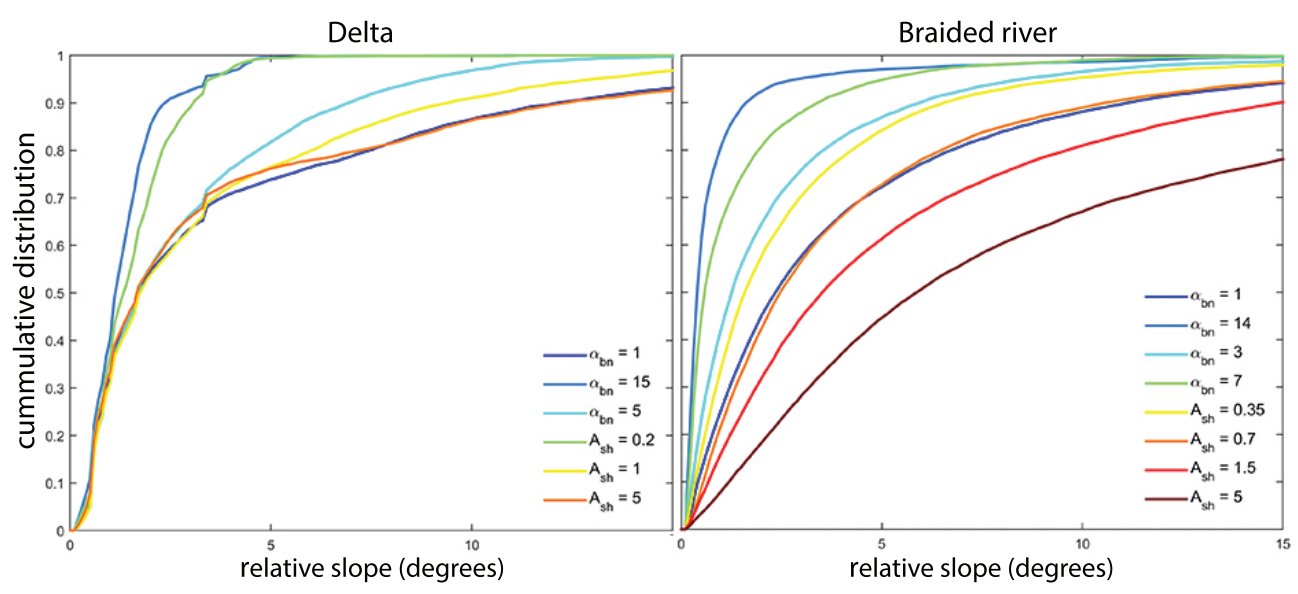

Figure 5. The relative distribution of transverse slopes in all models. Different lines represent different models with varying slope parameters.

\section{Implications}

The simple 5 grid cell wide model shows that different sediment transport predictors lead to a different balance between the transverse sediment transport and the channel incision. This implies that the choice of sediment transport predictor already influences the preference of the model to overdeepen channels, and thereby has a major influence on the large-scale morphodynamics.

The large-scale braided river model, with the sediment transport predictor of Engelund and Hansen [13], shows a larger difference between both slope options than the delta model, with the sediment transport predictor of Van Rijn et al. [12]. This difference in effect could partly be explained by the difference in sediment transport predictor, which influences the balance between incision and transverse sediment transport. Another large influence could be the modelled environment, since the braided river has a much larger braiding index and more bifurcations, and here the difference in sediment transport direction has a large influence by dividing sediment over bifurcates and balancing secondary currents.

The delta model furthermore shows that even though the amount of sediment transport is equal in one section, the effect on morphology could be significantly different in other sections of the model. This implies that calibrating large-scale morphology with different predictors could lead to different model outcomes due to the difference in direction of sediment transport on transverse slopes.

\section{References}

[1] F. Schuurman, W. A. Marra, and M. G. Kleinhans, Journal of Geophysical Research: Earth Surface 118(4), 2509-2527 (2013).

[2] N. Struiksma, K. W. Olesen, C. Flokstra, H. J. De Vriend, Journal of Hydraulic Research 23(1), 57-79 (1985).

[3] A. Crosato, E. Mosselman, Water Resources Research 45(3), 1-14 (2009).

[4] M. Bolla Pittaluga, G. Coco, M. G. Kleinhans, Geophysical Research Letters 42(18), 7521-7536 (2015). 
[5] S. J. Hulscher,Journal of Geophysical Research: Oceans 101(C9), 727-20,744 (1996).

[6] P. Blondeaux, G. Vittori, Continental Shelf Research 112, 31-45 (2016).

[7] M. Van der Wegen, J. A. Roelvink, Geomorphology 179, 152-167 (2012).

[8] A.W. Baar, J.C. De Smit, W.S.J. Uijttewaal, M.G. Kleinhans, Water Resources Research 54(1), 1944-7973 (2018)

[9] S. Ikeda, Journal of Hydraulic Engineering 110(2), 200-203 (1984).

[10] F. Koch, C. Flokstra, 19th International association for hydraulic research congress, (1981).

[11] L. C. Van Rijn, Principles of Sediment Transport in Rivers, Estuaries and Coastal Seas (Aqua Publications, the Netherlands, 1993).

[12] L. C. Van Rijn, D.J.R. Walstra, M. Van Ormondt, Deltares (WL) Z3748, (2004)

[13] F. Engelund, E. Hansen, A Monograph on Sediment Transport in Alluvial Streams, (Da. Tech. Press, Copenhagen, 1967). 